\title{
Haploidentical Hematopoietic Stem Cell Transplant Using Post-transplant Cyclophosphamide: A Single-Center Initial Experience in Vietnam
}

\author{
Man Van Huynh, Quang The Nguyen, Phu Duc Vinh Huynh, Nam Duy Hoang, Thu Hanh Nguyen, Dung Chi Phu
}

Stem Cell Transplantation Department, Blood Transfusion Hematology Hospital, Ho Chi Minh City, Vietnam

\begin{abstract}
Aims and objectives: Haploidentical transplants constitute a potential alternative therapy for patients who urgently need transplantation in the absence of human leukocyte antigen-matched donors. We report a singlecenter experience regarding the initial results of haploidentical peripheral blood stem cell transplantation (haplo-PBSCT) using post-transplant cyclophosphamide (PTCy) at the HCMC Blood Transfusion Hematology (BTH) Hospital.

Methods: We conducted a retrospective case series study of 23 patients who underwent haplo-PBSCT using PTCy at the HCMC BTH Hospital between January 2014 and January 2021. The refined disease risk index (DRI) was used to stratify the outcomes. We evaluated the engraftment rate, graft-versus-host disease (GVHD), and complications during haploidentical transplantation. Overall survival (OS), disease-free survival (DFS), and GVHD-free relapse-free survival (GRFS) were assessed.

Results: The majority of the patients in the present study were diagnosed with acute myeloid leukemia. All patients received reduced-intensity conditioning regimens. The engraftment rate was $86.9 \%$. The median times to neutrophil and platelet engraftment were 17 and 31 days, respectively. Two patients (8.7\%) reported severe acute GVHD (grade III-IV), while two patients (8.7\%) had grade I-II acute GVHD. Three patients experienced limited chronic GVHD of the skin, requiring topical steroids. The most common complication was bloodstream infection (60.9\%). Cytomegalovirus reactivation occurred in 19 patients $(82.6 \%)$ and $17.4 \%$ developed hemorrhagic cystitis. The 1-year relapse rate was 32.5\%. The cumulative incidence of non-relapse mortality at 1 year was $17.3 \%$. The 1-year OS and DFS rates were $66.3 \%$ and $55.7 \%$, respectively. The 1 -year GRFS rate was $49.2 \%$. A high/very high DRI score was associated with worse OS after haplo-PBSCT $(P=0.038)$.

Conclusion: Haploidentical transplant using PTCy is a feasible therapy for patients without suitably matched donors in Vietnam. Infection after transplantation remains a challenge and requires effective management.
\end{abstract}

Key words Cyclophosphamide, haploidentical transplantation, PTCy, vietnam

Submitted March 31, 2021; Accepted May 30, 2021; Published online November 25, 2021; Issued online November 25, 2021

Correspondence: Man Van Huynh, Stem Cell Transplantation Department, Blood Transfusion Hematology Hospital, 118 Hong Bang Street, Ward 12, District 5, Ho Chi Minh City, Vietnam, E-mail: huynhvanman@yahoo.com

\section{Introduction}

Hematopoietic stem cell transplantation (HSCT) is a potentially curative treatment for a wide variety of malignant and non-malignant hematological diseases. A human leukocyte antigen (HLA)-matched donor is the optimal option, leading to the best outcomes. However, HLA-matched siblings and unrelated donors are not always available, prompting various difficulties in treating patients with hematologic disorders and aggressive malignancies that require urgent HSCT.
The earliest attempts with haploidentical HSCT had significant problems due to high rates of graft rejection and severe acute graft-versus-host disease (GVHD) ${ }^{1}$. To overcome these disadvantages, experts from Johns Hopkins University initially evaluated the use of high-dose post-transplant cyclophosphamide (PTCy), which was shown to be an effective strategy with acceptable survival and low incidence of acute GVHD (aGVHD) and chronic GVHD (cGVHD) ${ }^{2}$. The role of PTCy has been extensively studied in recent years and has shown numerous promising outcomes ${ }^{3}$. Therefore, the number of 
haploidentical HSCT procedures has increased dramatically in many centers from the United States and Europe ${ }^{4}$.

A haploidentical donor could be diversely available among biological parents, siblings, aunts, uncles, and nephews. Traditional extended families are predominant in Vietnam and consist of many generations living together. Thus, it is relatively easy to find a donor for an HLA-haploidentical transplant. In addition, finding an unrelated donor is very challenging for many patients in Vietnam due to the lack of donor registries and financial constraints. Therefore, transplantation with haploidentical donors could be considered in developing countries, since it is reasonably cost-effective compared to other graft sources 5 .

The Blood Transfusion Hematology (BTH) Hospital has performed haploidentical peripheral blood hematopoietic stem cell transplantation (haplo-PBSCT) since 2013. We conducted this study to assess the preliminary results regarding the efficacy and complications of haplo-PBSCT using PTCy.

\section{Patients and Methods}

\section{Study design}

The present retrospective case series study included 23 patients who underwent haplo-PBSCT at the BTH Hospital, Hochiminh City, Vietnam between January 2014 and January 2021. HSCT was performed in both children and adults. The patients were diagnosed with malignant diseases such as acute myeloid leukemia (AML), acute lymphoblastic leukemia (ALL), myelodysplastic syndrome, and chronic myeloid leukemia. All patients were routinely followed-up at the hospital. The institutional review board of the BTH Hospital approved the protocol. In light of the retrospective medical record review, the requirement of informed consent was waived. This study was approved on 3 May 2020 (IRB approval 443/TMHH-HĐĐĐ) to be in accordance with the ethical standards and with the Declaration of Helsinki.

\section{Conditioning regimens, supportive care, and post- transplant follow-up}

Donors included HLA-haploidentical first-degree relatives. Anti-HLA antibodies were detected by panel reactive antibody bead-based testing using $\mathrm{LABScan}^{\mathrm{TM}}$ 100 Flow analyser (Luminex Corporation, Texas, USA). All patients received reduced-intensity conditioning (RIC) (Supplementary Table). The present study included three types of conditioning regimens. We developed a conditioning regimen of fludarabine and melphalan based on allogeneic stem cell transplantation in lymphoid neoplasms. Cytarabine was added to the regi- men for cytoreduction before conditioning ${ }^{6}$. The regimen of fludarabine, busulfan, and cyclophosphamide was developed based on myeloablative conditioning, as introduced by Solomon et al. ${ }^{7}$. Reduced-dose busulfan was modified to minimize toxicity. We combined thiotepa with fludarabine/busulfan to penetrate the blood-brain barrier and for antileukemic potential ${ }^{8}$.

High-dose cyclophosphamide (50 mg/kg/day) was administered on days +3 and +4 , which was followed by oral tacrolimus $(0.06 \mathrm{mg} / \mathrm{kg} /$ day) from day +5 (to reach a concentration of $5-10 \mathrm{ng} / \mathrm{mL}$ ) or intravenous cyclosporin $(3 \mathrm{mg} / \mathrm{kg} / \mathrm{day}$, to reach a concentration of 200-400 $\mathrm{ng} / \mathrm{mL})^{2}$. Calcineurin inhibitors were tapered from day +100 and completely stopped on day +180 unless GVHD occurred. In addition, mycophenolate mofetil (MMF) was administered at a dose of $15 \mathrm{mg} / \mathrm{kg}$ three times a day and discontinued on day +35 . Granulocyte colony-stimulating factor was administered from day +5 until absolute neutrophil count $($ ANC) $>0.5 \times$ $10^{9} / \mathrm{L}$ was achieved for two consecutive days. All patients received fluconazole, acyclovir, quinolone, and trimethoprim/sulfamethoxazole as prophylaxis for fungal, viral, bacterial, and Pneumocystis jiroveci infections. Cytomegalovirus (CMV) DNA level was checked via serum polymerase chain reaction (PCR) test once a week until day +100 . Bone marrow aspiration was performed on day +30 . Donor chimerism was evaluated on days $+30,+60,+90,+180$, and +365 .

\section{Endpoints and definitions}

The primary endpoints were overall survival (OS), disease-free survival (DFS), and GVHD-free relapsefree survival (GRFS). The secondary endpoints were neutrophil engraftment, platelet recovery, aGVHD, cGVHD, non-relapse mortality (NRM), relapse rate (RR), and significant complications after transplantation such as bacterial infections, CMV reactivation, and hemorrhagic cystitis. Neutrophil recovery time was defined as the time to first of the three consecutive days with an $\mathrm{ANC} \geq 0.5 \times 10^{\circ} / \mathrm{L}$. Platelet recovery time was defined as the time to first of the seven consecutive days with platelet count $\geq 20 \times 10^{9} / \mathrm{L}$ without platelet transfusion. We graded aGVHD according to the IBMTR Severity Index ${ }^{9}$ and cGVHD was graded according to the Revised Seattle Criteria ${ }^{10}$. OS was calculated until the date of death from any cause or the last follow-up visit. DFS was defined as the date of first relapse, death from any cause, or the last follow-up visit. GRFS was defined as the absence of grade III-IV aGVHD, cGVHD requiring systemic immunosuppressive treatment, disease relapse, or death from any cause during the first 12 and 24 months after haploidentical HSCT. 


\section{Statistical analysis}

OS and DFS were estimated using the Kaplan-Meier method. Univariate analyses were performed using logrank tests. Cumulative incidences of RR, NRM, engraftment, bacterial infections, GVHD, and CMV reactivation were estimated with competing-risk analysis using Gray's method. RR and NRM were considered mutually competing events. Death was treated as a competing event of engraftment and bacterial infections. Relapse or death without relapse or graft failure was considered as competing events of GVHD and CMV reactivation. The refined disease risk index (DRI) was used to stratify the outcomes ${ }^{11}$. Classification of patients based on disease risk status according to the DRI resulted in low-intermediate and high-very high groups. Statistical significance was set at $\mathrm{p}<0.05$. Statistical analysis was performed using SPSS version 13 (SPSS Inc., Chicago, IL, USA).

\section{Results}

\section{Patients and transplantation characteristics}

The baseline characteristics of the patients are summarized in Table 1. The median age at transplant was 40 years (range: 2-53 years). Most of the patients were men $(60 \%)$. The majority of the patients were diagnosed with AML. Twenty-two patients were in remission and had no history of HSCT before haploidentical transplantation. One patient diagnosed with ALL was refractory to chemotherapy before transplantation and underwent fludarabine, melphalan, and cytarabine conditioning regimen. The low-intermediate group based on DRI comprised of more than $70 \%$ of the patients. Additionally, all donors and patients were positive for CMV IgG antibody and negative for CMV IgM antibody. Among twenty patients testing for anti-HLA, one developed donor-specific anti-HLA antibodies. We used oral tacrolimus for most of the patients and intravenous cyclosporine in a 2-year-old girl who could not tolerate oral medication (Table 1).

\section{Engraftment}

Three patients died before engraftment due to septic shock. The engraftment rate was $86.9 \%$ (95\% confidence interval [CI]: 64.8-95.6\%), as confirmed by donor chimerism at day +30 after transplantation (Figure 1). The median times to neutrophil and platelet engraftment were 17 days (range: 14-23 days) and 31 days (range: 17-93 days), respectively. There were no records of primary graft failure or mixed chimerism. The patient who had a positive DSA donor was engrafted with complete donor chimerism.
Table 1. Patient characteristics

\begin{tabular}{|c|c|}
\hline Characteristics & Patients $(n=23)$ \\
\hline Median age (range) & $40(2-53)$ \\
\hline Sex (male:female) & $14: 9$ \\
\hline $\begin{array}{l}\text { Diagnosis, } \mathrm{n}(\%) \\
\text { AML } \\
C M L \\
\text { ALL } \\
\text { MDS }\end{array}$ & $\begin{aligned} 14 & (60.9) \\
2 & (8.7) \\
6 & (26.1) \\
1 & (4.3)\end{aligned}$ \\
\hline $\begin{array}{l}\text { HCT-Cl score, } \mathrm{n}(\%) \\
\quad \leq 1 \\
2\end{array}$ & $\begin{array}{c}22 \text { (95.7) } \\
1 \text { (4.3) }\end{array}$ \\
\hline $\begin{array}{l}\text { DRI score, n (\%) } \\
\text { Low - Intermediate } \\
\text { High - Very high }\end{array}$ & $\begin{array}{r}17(73.9) \\
6(26.1)\end{array}$ \\
\hline $\begin{array}{l}\text { Type of donors, n (\%) } \\
\text { Sibling } \\
\text { Father } \\
\text { Mother } \\
\text { Son } \\
\text { Daughter }\end{array}$ & $\begin{aligned} 12 & (52.2) \\
4 & (17.4) \\
1 & (4.3) \\
5 & (21.8) \\
1 & (4.3)\end{aligned}$ \\
\hline Donor - recipient sex mismatch, n (\%) & $10(43.4)$ \\
\hline $\begin{array}{l}\text { Blood group incompatibility, n (\%) } \\
\text { Major incompatibility } \\
\text { Minor incompatibility } \\
\text { Bidirectional incompatibility } \\
\text { Same blood group }\end{array}$ & $\begin{aligned} 1 & (4.3) \\
5 & (21.8) \\
1 & (4.3) \\
16 & (69.6)\end{aligned}$ \\
\hline $\begin{array}{l}\text { HLA matches, } n(\%) \\
5 / 10 \\
6 / 10 \\
7 / 10 \\
8 / 10\end{array}$ & $\begin{aligned} 14 & (60.9) \\
6 & (26.1) \\
2 & (8.7) \\
1 & (4.3)\end{aligned}$ \\
\hline $\begin{array}{l}\text { Anti-HLA testing, n (\%) } \\
\text { Positive DSA } \\
\text { Negative DSA }\end{array}$ & $\begin{array}{c}1(4.3) \\
19(82.6)\end{array}$ \\
\hline Median CD34+ (×10\%/kg) (range) & $7.17(5.7-8.11)$ \\
\hline $\begin{array}{l}\text { Conditioning regimen, } \mathrm{n}(\%) \\
\text { Flu/Bu/low dose } \mathrm{Cy} \\
\text { Flu/Bu/Thiotepa } \\
\text { Flu/Mel } \pm \text { Ara-C }\end{array}$ & $\begin{aligned} 16 & (69.6) \\
5 & (21.7) \\
2 & (8.7)\end{aligned}$ \\
\hline $\begin{array}{l}\text { Calcineurin inhibitors, } \mathrm{n}(\%) \\
\text { Tacrolimus } \\
\text { Cyclosporin }\end{array}$ & $\begin{aligned} 22 & (95.7) \\
1 & (4.3)\end{aligned}$ \\
\hline
\end{tabular}

Data are displayed in $\mathrm{n}(\%)$ for categorical variables and median (range) for continuous variables with non-normal distribution. ALL, acute lymphoblastic leukemia; AML, acute myeloid leukemia; Ara-C, arabinosylcytosine; Bu, busulfan; CML, chronic myeloid leukemia; Cy, cyclophosphamide; DSA, donor-specific anti-HLA antibodies; Flu, fludarabine; $\mathrm{HCT}-\mathrm{Cl}$, hematopoietic cell transplantation-specific comorbidity index; HLA, human leukocyte antigen; MDS, myelodysplastic syndrome; Mel, melphalan.

\section{Complications}

The estimated cumulative incidences of grade I-IV aGVHD and grade III-IV aGVHD on day +100 were $17.4 \%$ (95\% CI: $5.4-35 \%$ ) and $8.7 \%$ (95\% CI: $1.5-$ 24.2\%), respectively (Figure 2A). Two patients developed severe aGVHD of the skin, gastrointestinal tract, and liver. Systemic corticosteroid (prednisolone $1 \mathrm{mg}$ / $\mathrm{kg}$ per day) was initiated in these patients. Steroid doses were tapered for 21 days after resolution of the manifestations of aGVHD. Only three patients devel- 
oped limited cGVHD of the skin among the 19 patients who survived for more than 100 days after transplantation, accounting for $15.8 \%$ (95\% CI: 3.9-34.9\%) (Figure 2B). These patients appeared to respond to topical steroids for treating the symptoms of cGVHD.

Febrile neutropenia was observed in all enrolled patients. Bacterial bloodstream infection was documented at $60.9 \%$ (95\% CI: 38.3-77.4\%). Gram-negative bacterial isolates including Pseudomonas aeruginosa, Escherichia coli, and Klebsiella pneumonia were identified in most of the samples.

In our study, the cumulative incidence of CMV reactivation by day +100 after haplo-PBSCT was $82.6 \%$ (95\% CI: 60.1-93.1\%). The median time from transplant to reactivation was 26 days (range: 11-44 days). Patients with CMV reactivation underwent pre-emptive therapy with foscavir or ganciclovir. One patient exhibited secondary graft failure due to uncontrolled reactiva-

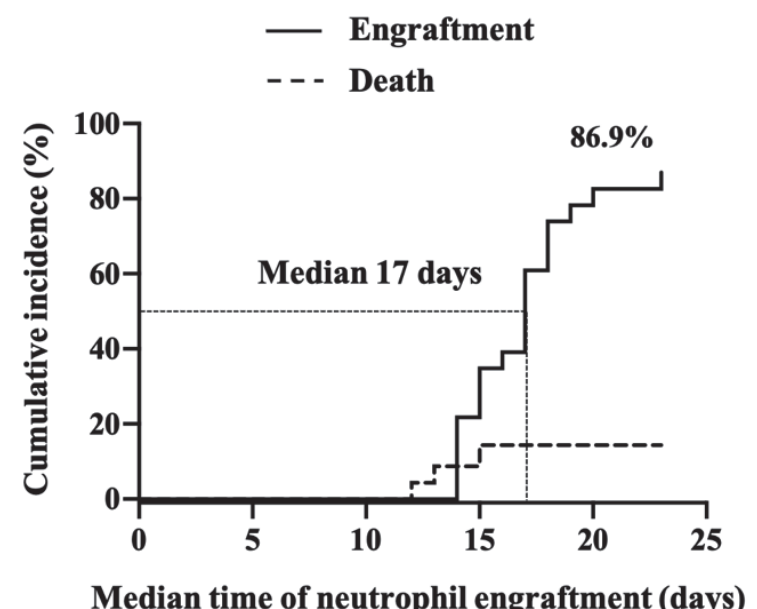

Figure 1. Cumulative incidences of neutrophil engraftment Death is the competing event of neutrophil engraftment. tion and ganciclovir toxicity. Four patients (17.4\%) developed grade II-III hemorrhagic cystitis and received hyperhydration, platelet transfusion, and hyperbaric oxygen therapy.

Altogether, seven patients died of both relapse and non-relapse-related causes (Table 2). The estimated cumulative incidence of 1-year NRM was $17.3 \%(95 \%$ confidence interval $[\mathrm{CI}]$ : 1.9-32.5\%) (Figure 3A). Multidrug-resistant Pseudomonas aeruginosa infection was the primary cause of death in three patients. One patient died of intracranial hemorrhage following refractory thrombocytopenia. The 1-year RR was $32.5 \%$ (95\% CI: $8.1-56.8 \%$ ) (Figure 3A). No statistically significant difference was observed in NRM and RR between the low-intermediate and the high-very high DRI groups.

\section{Survival}

The median follow-up for survivors was 12.27 months (range: 5.26-57.26 months). The 1-year OS and DFS rates were $66.3 \%$ (95\% CI: $45.3-87.3 \%)$ and $55.7 \%$ (95\% CI: 32.9-78.4\%), respectively (Figure 3B). Sixteen patients were alive at the last follow-up visit. The 1-year GRFS rate was 49.2\% (95\% CI: 27.4$70.9 \%$ ) (Figure 3B). The 1-year OS in low-intermediate DRI group tended to be better than that in the high-risk group $(78.4 \%$ vs. $33.3 \%, P=0.038$ ) (Figure $4 \mathrm{~A}$ and 4 B). However, no significant difference was observed in the 1-year DFS.

Table 2. Causes of death

\begin{tabular}{lc}
\hline \multicolumn{1}{c}{ Cause of death } & Patients $(\mathrm{n}=23)$ \\
\hline Relapse, $\mathrm{n}(\%)$ & $3(13 \%)$ \\
$\begin{array}{l}\text { Non-relapse, } \mathrm{n}(\%) \\
\text { Septic shock }\end{array}$ & $3(13 \%)$ \\
Intracranial hemorrhage & $1(4.3 \%)$ \\
\hline
\end{tabular}

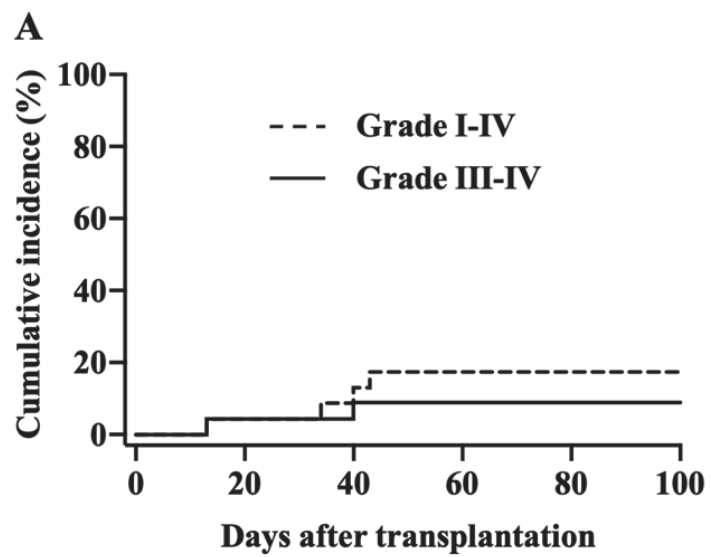

B

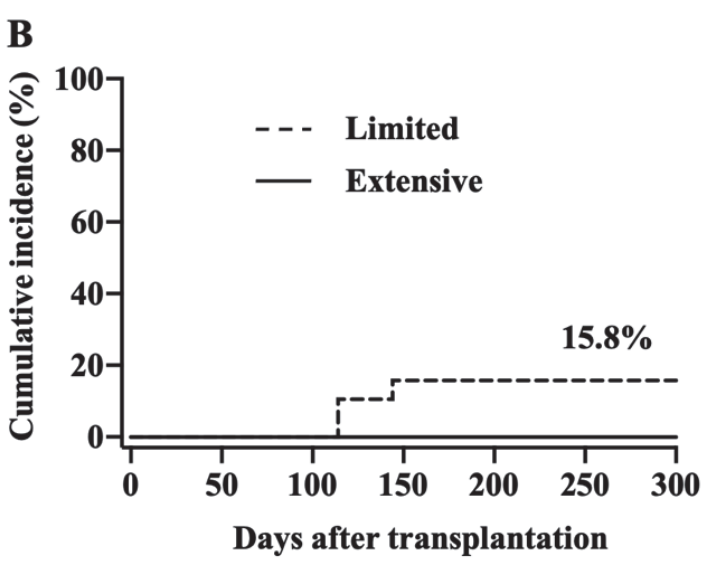

Figure 2. Cumulative incidences of $(A)$ acute graft-versus-host disease and (B) chronic graft-versus-host disease 

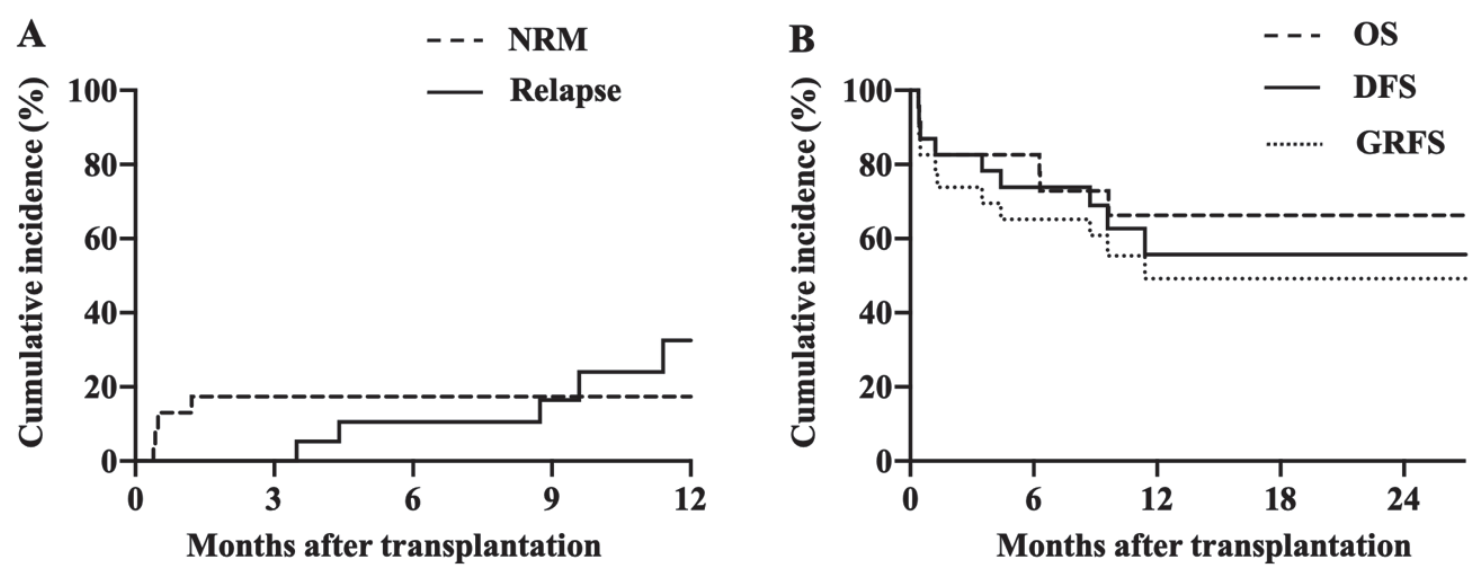

Figure 3. (A) Non-relapse mortality and relapse rate after haplo-PBSCT. (B) Overall survival, disease-free survival, and GVHD-free relapse-free survival after haplo-PBSCT

DFS, disease-free survival; NRM, non-relapse mortality; OS, overall survival.
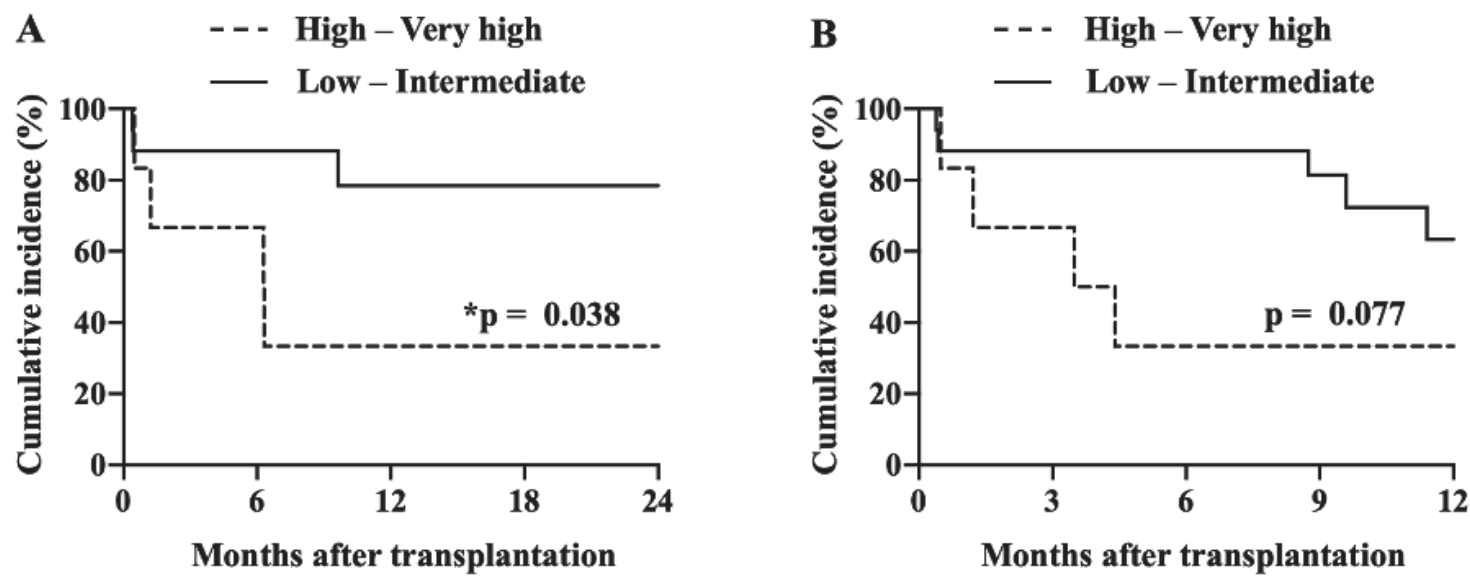

Figure 4. (A) 1-year overall survival by DRI group. (B) 1-year disease-free survival by DRI group

\section{Discussion}

The present study has reported our initial experience on haploidentical transplantation with high-dose cyclophosphamide for GVHD prophylaxis. Our results showed that haplo-PBSCT using PTCy resulted in a high rate of engraftment (86.9\%). Several studies based on haplo-PBSCT with PTCy using peripheral blood stem cells have been conducted worldwide ${ }^{12-14}$. These reports exhibited good donor cell engraftment rate $(87 \%$ to $100 \%)$. The neutrophil recovery time in our patients was similar to that reported in previously published reports (17 to 20 days). Raj et al. performed a multicenter study on haplo-PBSCT with PTCy using peripheral blood stem cells in 55 high-risk patients ${ }^{14}$. The results showed that neutrophil and platelet recovery times were 17 and 21 days, respectively. In our study, the median platelet recovery time was relatively longer (31 days).

It appears that management of GVHD complications in both bone marrow and peripheral blood graft sources benefited from post-transplant high-dose cyclophosphamide ${ }^{14-18}$. In our study, two patients developed severe aGVHD involving the skin, gut, and liver. All patients responded to steroids at a dose of $1 \mathrm{mg} / \mathrm{kg}$. The low incidence of severe aGVHD in PTCy-based transplantation might be due to selective elimination and clonal deletion of alloreactive $\mathrm{T}$ cells ${ }^{19}$. The cumulative incidence of limited cGVHD at 1 year was $15.8 \%$. Notably, extensive cGVHD was not observed. The rate of cGVHD observed in several published studies was also significantly lower in haploidentical transplantation using PTCy when compared with matched related donor (MRD) and matched unrelated donor (MUD) transplantations $^{18,20,21}$. Therefore, the advantage of PTCy combined with MMF and calcineurin inhibitor in haploidentical HSCT for the prophylaxis of acute and chronic GVHD was notable, which in turn improved the quality of life of patients after transplantation.

The 1-year OS and DFS rates in our study were encouraging. Other studies on haplo-PBSCT have also demonstrated similar OS and DFS rates ${ }^{12-14,17}$. In 2013, 
Bashley et al. performed a study comparing haploidentical HSCT using PTCy $(n=116)$ with MRD $(n=181)$ and MUD $(n=178)$ transplantation. The 2-year OS in haploidentical HSCT with PTCy was $64 \%$ and the 2year OS rates in matched MRD and MUD transplantations were $76 \%$ and $67 \%$, respectively with no statistically significant difference ${ }^{22}$. These results suggest that haplo-PBSCT with PTCy might be an effective alternative option in patients in the absence of fully HLAmatched donors. Recent studies have proposed a novel composite endpoint of GRFS to evaluate clinical outcomes, since OS alone cannot fully reflect the longlasting complications after transplantation ${ }^{23,24}$. In 2020, Mehta et al. reported that haploidentical transplantation offered superior GRFS among patients using RIC when compared with cord blood and bone marrow stem cell transplantation $^{25}$. Hence, combining GRFS and OS in future studies could provide more information to analyze the efficacy of haploidentical transplantation in Vietnam.

With a median follow-up of 12.27 months, the estimated cumulative incidence of NRM at 1-year was $17.3 \%$ in our study. Most of the non-relapse-related deaths are associated with severe bacterial infections. Bashley et al. showed that the NRM of haplo-PBSCT using PTCy was significantly lower than that of MRD/ MUD-HSCT $(P=0.02)^{22}$. Besides, other studies have also indicated no difference in the 1-year NRM between haplo-PBSCT using PTCy (with both myeloablative conditioning [MAC] and RIC) and MRD/MUD$\mathrm{HSCT}^{18,19,21,26}$. Hence, these findings suggest the effectiveness of haploidentical HSCT with PTCy on nonrelapse mortality in both MAC and RIC conditioning regimens.

Despite acceptable NRM, bacterial infection has been a significant source of morbidity and mortality in haploidentical transplantation in Vietnam. Most of the isolated bacteria were gram-negative bacilli. In a retrospective cohort study on haplo-PBSCT using PTCy, Slade et al. reported a comparable number of patients who experienced bacterial infections $(62 \%)$. In the aforementioned study, organisms causing bacterial bloodstream infections included Enterococcus species, Pseudomonas aeruginosa, and other gram-negative bacilli ${ }^{27}$. In 2015, Yan et al. conducted a study in China to analyze the causes of mortality after haploidentical hematopoietic stem cell transplantation ${ }^{28}$. The results indicated that infection was the leading cause of death in transplantation. The mortality due to infection in haploidentical HSCT was significantly higher than that in fully matched HLA transplant, with rates of $21.2 \%$ and $13.4 \%$, respectively $(P=0.002)$. Therefore, practical strategies to optimize antibiotic utility based on specific local pathogens are important during haploidentical transplantation, especially in limited-resource countries.

Although prevalence of CMV-exposed population in Vietnam has not been published, all the donors and patients in our study were positive for CMV IgG. Thus, the patients were considered at high risk of reactivation, which explains the high incidence of $\mathrm{CMV}$ reactivation $(82.6 \%)$ in quantitative $\mathrm{PCR}^{29}$. Although letermovir has proven its effectiveness in preventing CMV infection in CMV-seropositive patients undergoing haploidentical HSCT using PTCy, it is not yet available in Vietnam ${ }^{30}$. Our previously published data suggested that $76.9 \%$ of the patients who underwent HLA-matched allogeneic stem cell transplantation also exhibited CMV reactivation $^{31}$. Many investigations on haploidentical transplants have reported a broad range of incidence of CMV reactivation $(26 \% \text { to } 55 \%)^{2,12,27,32-34}$. A possible explanation for this variation might be the lack of consensus regarding the threshold of CMV DNA to define a positive status. Slow immune reconstitution in haploidentical HSCT using PTCy might result in higher rates of viral and bacterial infections when compared with fully matched HSCT $^{12}$. Recent studies have suggested that CMV reactivation is associated with protection from leukemic relapse, especially in $\mathrm{AML}^{35-38}$. However, ganciclovir treatment is also a concern in haploidentical transplantation. In our study, both ganciclovir toxicity and uncontrolled CMV reactivation were responsible for secondary graft failure in one patient. A study by Akahoshi et al. involving 184 HSCT patients who achieved platelet recovery also demonstrated that the use of ganciclovir or valganciclovir increased the risk of secondary thrombocytopenia after transplantation. Recent studies and ongoing clinical trials for immunotherapy targeting specific cytotoxic $\mathrm{T}$ cells have contributed to further advances in the management of refractory CMV reactivation $^{39-41}$.

According to a study by Baskett et al., the incidence of BK virus hemorrhagic cystitis in the haploidentical HSCT-PTCy platform was $19 \%{ }^{42}$. However, one of the limitations of our study was the inability to analyze urine BK samples. The incidence of hemorrhagic cystitis was $17.4 \%$. These patients also had CMV reactivation. In studies on haploidentical transplantation, the incidence of hemorrhagic cystitis has been quite diverse, ranging from $7 \%$ to $40 \%{ }^{12,27,32-34}$. Acrolein, a metabolite of cyclophosphamide, is also one of the causes of hemorrhagic cystitis. In 2015, a study also showed that cyclophosphamide and reactivation of CMV in haploidentical transplant were risk factors for increased rates of hemorrhagic cystitis $(P=0.05)^{43}$. Therefore, monitoring BK viral load and immune recovery should be considered in this complication of haploidentical HSCT using PTCy.

The DRI is a validated tool used to categorize the 
risk of impacting survival based on the disease and pretransplant conditions. Armand et al. demonstrated a statistically significant difference in survival between the groups $(P<0.0001)^{11}$. After analyzing the correlation between DRI and OS in our study, the group of patients with high to very high DRI had significantly lower 1year OS than those with low-intermediate DRI. Similarly, McCurdy et al. demonstrated that OS and progression-free survival of the high-very high DRI group were notably lower than those of the lowintermediate group ${ }^{18}$. These findings demonstrate the effectiveness of DRI in pre-transplant risk assessment to predict transplantation outcomes. Moreover, it might assist in proposing a strategy to improve haploidentical transplant results with a novel maintenance therapy. A recent study by Sugita et al. also reported a relapse rate of $0 \%{ }^{17}$. The authors suggested that haploidentical HSCT using PTCy after RIC might show promising results in reducing the relapse rate in the lowintermediate DRI group, providing thoroughly positive benefits.

In conclusion, haplo-PBSCT with PTCy provided the benefit of reducing the time to find suitable donors in patients who need early disease control. Moreover, the initially analyzed 1-year OS and DFS rates were promising. The present study also indicated that complications such as infection after haploidentical HSCT are challenging and need to be managed carefully. Although this is the first report of haploidentical HSCT in Vietnam, our study examined only a small group of patients. Further research should be conducted in the future to determine the long-term benefits in the Vietnamese population.

\section{Acknowledgments}

We would like to thank the medical staff at the Stem Cell Transplantation Department of the Blood Transfusion Hematology Hospital for taking care of the patients.

\section{Author Contributions}

M.V.H, Q.T.N, P.D.V.H, and D.C.P performed the study design.

M.V.H, Q.T.N, and P.D.V.H wrote the manuscript.

T.H.N, Q.T.N, and N.D.H reported and analyzed data.

D.C.P supervised the manuscript.

\section{Conflicts of Interest}

The authors declare no conflict of interest. Disclosure forms provided by the authors are available on the web- site.

\section{References}

1. Powles R. L., Morgenstern G. R., Kay H. E., McElwain T. J., Clink H. M., Dady P. J., et al. Mismatched family donors for bone-marrow transplantation as treatment for acute leukaemia. Lancet. 1983; 1: 612-5.

2. Luznik L., O'Donnell P. V., Symons H. J., Chen A. R., Leffell M. S., Zahurak M., et al. HLA-haploidentical bone marrow transplantation for hematologic malignancies using nonmyeloablative conditioning and high-dose, posttransplantation cyclophosphamide. Biol Blood Marrow Transplant. 2008; 14: 641-50.

3. McCurdy S. R., Kasamon Y. L., Kanakry C. G., BolanosMeade J., Tsai H. L., Showel M. M., et al. Comparable composite endpoints after HLA-matched and HLA-haploidentical transplantation with post-transplantation cyclophosphamide. Haematologica. 2017; 102: 391-400.

4. Lee C. J., Savani B. N., Mohty M., Labopin M., Ruggeri A., Schmid C., et al. Haploidentical hematopoietic cell transplantation for adult acute myeloid leukemia: a position statement from the Acute Leukemia Working Party of the European Society for Blood and Marrow Transplantation. Haematologica. 2017; 102: 1810-22.

5. Fuchs E. J. Related haploidentical donors are a better choice than matched unrelated donors: Point. Blood Adv. 2017; 1: $397-400$

6. Anderlini P., Saliba R., Acholonu S., Giralt S. A., Andersson B., Ueno N. T., et al. Fludarabine-melphalan as a preparative regimen for reduced-intensity conditioning allogeneic stem cell transplantation in relapsed and refractory/Hodgkin's lymphoma: the updated M.D. Anderson Cancer Center experience. Haematologica. 2008; 93: 257-64.

7. Solomon S. R., Sizemore C. A., Sanacore M., Zhang X., Brown S., Holland H. K., et al. Haploidentical transplantation using $\mathrm{T}$ cell replete peripheral blood stem cells and myeloablative conditioning in patients with high-risk hematologic malignancies who lack conventional donors is well tolerated and produces excellent relapse-free survival: results of a prospective phase II trial. Biol Blood Marrow Transplant. 2012; 18: 1859-66.

8. Pagliardini T., Castagna L., Harbi S., Porta M. D., Rey J., Furst S., et al. Thiotepa, Fludarabine, and Busulfan Conditioning Regimen before T Cell-Replete Haploidentical Transplantation with Post-Transplant Cyclophosphamide for Acute Myeloid Leukemia: A Bicentric Experience of 100 Patients. Biol Blood Marrow Transplant. 2019; 25: 1803-9.

9. Rowlings P. A., Przepiorka D., Klein J. P., Gale R. P., Passweg J. R., Henslee-Downey P. J., et al. IBMTR Severity Index for grading acute graft-versus-host disease: retrospective comparison with Glucksberg grade. Br J Haematol. 1997; 97: 855-64.

10. Lee S. J., Vogelsang G., Flowers M. E. Chronic graft-versushost disease. Biol Blood Marrow Transplant. 2003; 9: 215-33.

11. Armand P., Kim H. T., Logan B. R., Wang Z., Alyea E. P., Kalaycio M. E., et al. Validation and refinement of the Disease Risk Index for allogeneic stem cell transplantation. Blood. 2014; 123: 3664-71.

12. Castagna L., Crocchiolo R., Furst S., Bramanti S., El Cheikh J., Sarina B., et al. Bone marrow compared with peripheral 
blood stem cells for haploidentical transplantation with a nonmyeloablative conditioning regimen and post-transplantation cyclophosphamide. Biol Blood Marrow Transplant. 2014; 20: 724-9.

13. Cieri N., Greco R., Crucitti L., Morelli M., Giglio F., Levati G., et al. Post-transplantation Cyclophosphamide and Sirolimus after Haploidentical Hematopoietic Stem Cell Transplantation Using a Treosulfan-based Myeloablative Conditioning and Peripheral Blood Stem Cells. Biol Blood Marrow Transplant. 2015; 21: 1506-14.

14. Raj K., Pagliuca A., Bradstock K., Noriega V., Potter V., Streetly M., et al. Peripheral blood hematopoietic stem cells for transplantation of hematological diseases from related, haploidentical donors after reduced-intensity conditioning. Biol Blood Marrow Transplant. 2014; 20: 890-5.

15. Bacigalupo A., Dominietto A., Ghiso A., Di Grazia C., Lamparelli T., Gualandi F., et al. Unmanipulated haploidentical bone marrow transplantation and post-transplant cyclophosphamide for hematologic malignanices following a myeloablative conditioning: an update. Bone Marrow Transplant. 2015; 50 Suppl 2: S37-9.

16. Grosso D., Gaballa S., Alpdogan O., Carabasi M., FilickoO'Hara J., Kasner M., et al. A two-step approach to myeloablative haploidentical transplantation: low nonrelapse mortality and high survival confirmed in patients with earlier stage disease. Biol Blood Marrow Transplant. 2015; 21: 646-52.

17. Sugita J., Kawashima N., Fujisaki T., Kakihana K., Ota S., Matsuo K., et al. HLA-Haploidentical Peripheral Blood Stem Cell Transplantation with Post-Transplant Cyclophosphamide after Busulfan-Containing Reduced-Intensity Conditioning. Biol Blood Marrow Transplant. 2015; 21: 1646-52.

18. McCurdy S. R., Kanakry J. A., Showel M. M., Tsai H. L., Bolanos-Meade J., Rosner G. L., et al. Risk-stratified outcomes of nonmyeloablative HLA-haploidentical BMT with high-dose posttransplantation cyclophosphamide. Blood. 2015; 125: 3024-31.

19. Ciurea S. O., Zhang M. J., Bacigalupo A. A., Bashey A., Appelbaum F. R., Aljitawi O. S., et al. Haploidentical transplant with posttransplant cyclophosphamide vs matched unrelated donor transplant for acute myeloid leukemia. Blood. 2015; 126: $1033-40$.

20. Garciaz S., Castagna L., Bouabdallah R., Furst S., Bramanti S., Coso D., et al. Familial haploidentical challenging unrelated donor Allo-SCT in advanced non-Hodgkin lymphomas when matched related donor is not available. Bone Marrow Transplant. 2015; 50: 865-7.

21. McCurdy S. R., Fuchs E. J. Comparable Outcomes for Hematologic Malignancies after HLA-Haploidentical Transplantation with Posttransplantation Cyclophosphamide and HLAMatched Transplantation. Adv Hematol. 2015; 2015: 431923.

22. Bashey A., Zhang X., Jackson K., Brown S., Ridgeway M., Solh M., et al. Comparison of Outcomes of Hematopoietic Cell Transplants from T-Replete Haploidentical Donors Using Post-Transplantation Cyclophosphamide with 10 of 10 HLAA, -B, -C, -DRB1, and -DQB1 Allele-Matched Unrelated Donors and HLA-Identical Sibling Donors: A Multivariable Analysis Including Disease Risk Index. Biol Blood Marrow Transplant. 2016; 22: 125-33.

23. Holtan S. G., DeFor T. E., Lazaryan A., Bejanyan N., Arora M., Brunstein C. G., et al. Composite end point of graftversus-host disease-free, relapse-free survival after allogeneic hematopoietic cell transplantation. Blood. 2015; 125: 1333-8.

24. Liu Y. C., Chien S. H., Fan N. W., Hu M. H., Gau J. P., Liu C. J., et al. Prognostic Factors on the Graft-versus-Host Disease-Free and Relapse-Free Survival after Adult Allogeneic Hematopoietic Stem Cell Transplantation. Stem Cells Int. 2016; 2016: 5143071.

25. Mehta R. S., Holtan S. G., Wang T., Hemmer M. T., Spellman S. R., Arora M., et al. Composite GRFS and CRFS Outcomes After Adult Alternative Donor HCT. J Clin Oncol. 2020; 38: 2062-76.

26. Raiola A. M., Dominietto A., di Grazia C., Lamparelli T., Gualandi F., Ibatici A., et al. Unmanipulated haploidentical transplants compared with other alternative donors and matched sibling grafts. Biol Blood Marrow Transplant. 2014; 20: $1573-9$.

27. Slade M., Goldsmith S., Romee R., DiPersio J. F., Dubberke E. R., Westervelt P., et al. Epidemiology of infections following haploidentical peripheral blood hematopoietic cell transplantation. Transpl Infect Dis. 2017; 19.

28. Yan C. H., Xu L. P., Wang F. R., Chen H., Han W., Wang Y., et al. Causes of mortality after haploidentical hematopoietic stem cell transplantation and the comparison with HLAidentical sibling hematopoietic stem cell transplantation. Bone Marrow Transplant. 2016; 51: 391-7.

29. Styczynski J. Who Is the Patient at Risk of CMV Recurrence: A Review of the Current Scientific Evidence with a Focus on Hematopoietic Cell Transplantation. Infect Dis Ther. 2018; 7: 1-16.

30. Lin A., Flynn J., DeRespiris L., Figgins B., Griffin M., Lau C., et al. Letermovir for Prevention of Cytomegalovirus Reactivation in Haploidentical and Mismatched Adult Donor Allogeneic Hematopoietic Cell Transplantation with PostTransplantation Cyclophosphamide for Graft-versus-Host Disease Prophylaxis. Transplant Cell Ther. 2021; 27: 85 e1-6.

31. Phu DVH, Man VH, Nam DH, Ngon TH, Tuan XM, Thu $\mathrm{HN}$, et al. Hematopoietic Stem Cell Transplantation in Vietnam: Current Status and Future Directions. Journal of Pharmacy and Pharmacology. 2018; 6.

32. Ciurea S. O., Mulanovich V., Saliba R. M., Bayraktar U. D., Jiang Y., Bassett R., et al. Improved early outcomes using a T cell replete graft compared with $\mathrm{T}$ cell depleted haploidentical hematopoietic stem cell transplantation. Biol Blood Marrow Transplant. 2012; 18: 1835-44.

33. Fayard A., Daguenet E., Blaise D., Chevallier P., Labussiere H., Berceanu A., et al. Evaluation of infectious complications after haploidentical hematopoietic stem cell transplantation with post-transplant cyclophosphamide following reducedintensity and myeloablative conditioning: a study on behalf of the Francophone Society of Stem Cell Transplantation and Cellular Therapy (SFGM-TC). Bone Marrow Transplant. 2019; 54: 1586-94.

34. Raiola A. M., Dominietto A., Ghiso A., Di Grazia C., Lamparelli T., Gualandi F., et al. Unmanipulated haploidentical bone marrow transplantation and posttransplantation cyclophosphamide for hematologic malignancies after myeloablative conditioning. Biol Blood Marrow Transplant. 2013; 19: 117-22.

35. Litjens N. H. R., van der Wagen L., Kuball J., Kwekkeboom J. Potential Beneficial Effects of Cytomegalovirus Infection after Transplantation. Front Immunol. 2018; 9: 389.

36. Yoon J. H., Lee S., Kim H. J., Jeon Y. W., Lee S. E., Cho B. 
S., et al. Impact of cytomegalovirus reactivation on relapse and survival in patients with acute leukemia who received allogeneic hematopoietic stem cell transplantation in first remission. Oncotarget. 2016; 7: 17230-41.

37. Elmaagacli A. H., Koldehoff M. Cytomegalovirus replication reduces the relapse incidence in patients with acute myeloid leukemia. Blood. 2016; 128: 456-9.

38. Jang J. E., Kim S. J., Cheong J. W., Hyun S. Y., Kim Y. D., Kim Y. R., et al. Early CMV replication and subsequent chronic GVHD have a significant anti-leukemic effect after allogeneic HSCT in acute myeloid leukemia. Ann Hematol. 2015; 94: 275-82.

39. Cho S. Y., Lee D. G., Kim H. J. Cytomegalovirus Infections after Hematopoietic Stem Cell Transplantation: Current Status and Future Immunotherapy. Int J Mol Sci. 2019; 20

40. Neuenhahn M., Albrecht J., Odendahl M., Schlott F., Dossinger G., Schiemann M., et al. Transfer of minimally manipulated CMV-specific $\mathrm{T}$ cells from stem cell or third-party donors to treat CMV infection after allo-HSCT. Leukemia. 2017; 31: 2161-71.

41. Pei X. Y., Zhao X. Y., Chang Y. J., Liu J., Xu L. P., Wang Y., et al. Cytomegalovirus-Specific T-Cell Transfer for Refractory Cytomegalovirus Infection After Haploidentical Stem Cell Transplantation: The Quantitative and Qualitative Immune Recovery for Cytomegalovirus. J Infect Dis. 2017; 216: 94556.

42. Baskett J., Culos K. A., Satyanarayana G., Patel D., Engelhardt B., Savani B., et al. Risk factors associated with early viral reactivation following haploidentical hematopoietic cell transplantation with post-transplant cyclophosphamide: a pilot study. Ann Hematol. 2020; 99: 1137-9.

43. Ruggeri A., Roth-Guepin G., Battipaglia G., Mamez A. C., Malard F., Gomez A., et al. Incidence and risk factors for hemorrhagic cystitis in unmanipulated haploidentical transplant recipients. Transpl Infect Dis. 2015; 17: 822-30.

https://doi.org/10.31547/bct-2021-002

Copyright (C)2021 Asia-Pacific Blood and Marrow Transplantation Group (APBMT). This is an open access article distributed under CC BY-NC license (https://creativecommon s.org/licenses/by-nc/4.0/). 